\title{
BETTI NUMBERS FOR NUMERICAL SEMIGROUP RINGS
}

\author{
DUMITRU I. STAMATE
}

\begin{abstract}
We survey results related to the magnitude of the Betti numbers of numerical semigroup rings and of their tangent cones.
\end{abstract}

\section{InTRODUCTION}

A numerical semigroup $H$ is a subset of the set of nonnegative integers $\mathbb{N}$, closed under addition, containing 0 (i.e. a monoid) and such that $|\mathbb{N} \backslash H|<\infty$. The latter condition may be replaced by having $\operatorname{gcd}(h: h \in H)=1$. The largest integer not in the numerical semigroup $H$ is called its Frobenius number, which we denote $F(H)$.

Given the positive integers $a_{1}, \ldots, a_{n}$, the monoid they generate is

$$
\left\langle a_{1}, \ldots, a_{n}\right\rangle=\left\{\sum_{i=1}^{n} k_{i} a_{i}: k_{i} \in \mathbb{N}, i=1, \ldots, n\right\} .
$$

Let $d=\operatorname{gcd}\left(a_{1}, \ldots, a_{n}\right)$. Since the semigroups $\left\langle a_{1}, \ldots, a_{n}\right\rangle$ and $\left\langle a_{1} / d, \ldots, a_{n} / d\right\rangle$ are isomorphic, it is clear that the study of submonoids of $\mathbb{N}$ reduces to the study of numerical semigroups. It is easy to check that any monoid $H \subset \mathbb{N}$ is finitely generated and that it has a unique minimal generating set that we denote $G(H)$. The embedding dimension of $H$ is defined as $\operatorname{emb} \operatorname{dim}(H)=|G(H)|$, the multiplicity of $H$ is $\mathrm{m}(H)=\min G(H)$ and its width is $\operatorname{wd}(H)=\max G(H)-\min G(H)$.

Unless otherwise stated, throughout this paper $H$ denotes a numerical semigroup and any semigroup is assumed to be a numerical semigroup.

Let $K$ be any field. The semigroup ring $K[H]$ is the $K$-subalgebra of the polynomial ring $K[t]$ generated by the monomials $t^{h}$ where $h \in H$. Assume $G(H)=$ $\left\{a_{1}, \ldots, a_{n}\right\}$. We consider the polynomial ring $S=K\left[x_{1}, \ldots, x_{n}\right]$ endowed with the nonstandard grading induced by $H$, namely $\operatorname{deg} x_{i}=a_{i}$ for all $i$. Then the $K$-algebra map $\varphi: S \rightarrow K[H]$ letting $\varphi\left(x_{i}\right)=t^{a_{i}}$ is a graded map. Its kernel $I_{H}=\operatorname{ker} \varphi$ is also called the toric ideal of $H$ since it is the ideal of relations of the toric algebra $K[H]$. More generally, any 1-dimensional toric ring can be viewed as a semigroup ring $K[H]$ with $H$ a numerical semigroup.

The elements of any minimal set of (homogeneous) generators of $I_{H}$ are the first syzygies of $K[H]$, and their count is the first Betti number of $K[H]$, denoted $\beta_{1}(K[H])$. A minimal set of relations among the first syzygies consists of what are called the second syzygies of $K[H]$, which are counted by $\beta_{2}(K[H])$. The process

2010 Mathematics Subject Classification. Primary 13-02, 13D02, 13A30, 16S36 ; Secondary $13 \mathrm{H} 10,13 \mathrm{P} 10$.

Key words and phrases. numerical semigroup rings, tangent cones, free resolution, Betti numbers, (almost) symmetric semigroup, shifted families of semigroups. 
continues, and it produces nontrivial syzygies until we reach the projective dimension of $K[H]$. The Betti sequence of $K[H]$ is the list $\left(\beta_{0}(K[H]), \beta_{1}(K[H]), \ldots\right)$, where $\beta_{0}(K[H])=1$. The last nonzero Betti number, namely $\beta_{n-1}(K[H])$ is called the (Cohen-Macaulay) type of $K[H]$ (or of the semigroup $H$, see also Section 3 ).

An algebra that is closely related to $K[H]$ and is relevant to our study is its associated graded ring with respect to the maximal ideal $\mathfrak{m}=\left(t^{h}: h \in H \backslash\{0\}\right) K[H]$, i.e. $\operatorname{gr}_{\mathfrak{m}} K[H]=\oplus_{i \geq 0} \mathfrak{m}^{i} / \mathfrak{m}^{i+1}$. This algebra is also called the tangent cone of $K[H]$ (or of $H$ ) in resemblance with the geometric origin of the concept, see [24, Chapter $5]$.

Minimal free resolutions of modules and their attached invariants are a classical and nevertheless constant source of questions in algebraic geometry and in commutative algebra, see [25], [60]. In this survey we collect some of the results and questions pertaining to these topics when the modules are $K[H]$ or $\operatorname{gr}_{\mathfrak{m}} K[H]$ for a numerical semigroup $H$. It is clear that general results may be also applied to this setting, but on the other hand new tools become available and we get sharper statements when we focus on the type of rings we mentioned.

We briefly outline the structure of this paper. In Section 2 we discuss arithmetic consequences (and at times characterizations) of the Gorenstein, complete intersection or Cohen-Macaulay property for $K[H]$ and $\operatorname{gr}_{\mathfrak{m}} K[H]$. We present how to start the resolution of these algebras, namely how to (algorithmically) compute the toric ideal $I_{H}$ and its ideal of initial forms $I_{H}^{*}$. Exact, but somehow opaque, formulas for the Betti numbers of $K[H]$ are given in terms of topological and combinatorial data encoded in the squarefree divisor complexes of the elements in the semigroup, introduced in [13].

Fröberg, Gottlieb and Häggkvist [28] defined the type of the semigroup $H$ as the cardinality of its set of its pseudo-Frobenius numbers: $P F(H)=\{x \in \mathbb{Z} \backslash H$ : $x+h \in H$ for all $0 \neq h \in H\}$. In Section 3 we give a detailed proof of the fact that this notion of type coincides with the Cohen-Macaulay type of the semigroup ring $K[H]$. In higher embedding dimension the type is also unbounded, but we present estimates for it when $H$ is in certain families of semigroups.

A recent result of $\mathrm{Vu}[74]$ is that if we bound the width of the semigroup, then the Betti numbers of $K[H]$ are bounded, too. This was extended to the Betti numbers of $\operatorname{gr}_{\mathfrak{m}} K[H]$ by Herzog and the present author in [41]. These are consequences of an eventual periodic behaviour of the Betti sequence of the toric ring and its tangent cone for semigroups in the same shifted family. We collect in Section 4 the most important periodicity-like results that have been spotted so far in shifted families of semigroups. There is a growing interest in this topic, due to possible applications in faster implementations of the known algorithms for computing invariants of $K[H]$ or $H$, see [18] and [57].

We say that the semigroup $H$ is obtained from the semigroup $L$ by a simple gluing if $H=\langle c L, \ell\rangle$ with $c, \ell>1$ coprime and $\ell \in L \backslash G(L)$. In Section 5 we explain how the Betti numbers of $K[L]$ and $K[H]$ are related; in particular they have the same type. 
In Section 6 we discuss two families of semigroups for whom the whole resolution of the associated toric ring is known: the complete intersections and those generated by an arithmetic sequence. It has been known since Herzog's [37] that at most three binomials suffice to generate $I_{H}$ when $H$ is 3-generated. On the other hand, it is possible in this embedding dimension that $I_{H}^{*}$ have as many generators as one wants, see Section 7.

Bresinsky [11] produced the first examples of 4-generated semigroups where $\mu\left(I_{H}\right)$ is arbitrarily large. In Section 8 we give a detailed proof of the computation of the Betti sequence of $K[H]$ and $\operatorname{gr}_{\mathfrak{m}} K[H]$ when $H$ is of Bresinsky's type, and also in a related family found by Arslan [3].

For several families of 4-generated semigroups the resolution of their toric ring is known, and we present these in Section 9. Namely, when $H$ is symmetric, pseudosymmetric or almost symmetric, or when it is generated by an almost arithmetic sequence. It is still obscure and not yet completed (or even started) the list of possible Betti sequences of $\operatorname{gr}_{\mathfrak{m}} K[H]$ when $H$ is in one of the said families.

For the background and the undefined terminology from commutative algebra we refer to the monographs [24], [14], and for an introduction to numerical semigroups to [64] and [61]. The lecture notes of Fröberg [29] from the conference in Porto in 2008 may also serve as an introduction to the topics we present. Resolutions and toric ideals are rarely computed by hand, and we are happy that software like Singular [20], Macaulay2 [36] or CoCoA [1] exists. In Singular, the package toric.1ib implements several algorithms for computing toric ideals, which are particularly efficient for numerical semigroup rings since these are not standard graded. Presentations for numerical semigroups can also be computed via the numericalsgps package [21] in GAP [30] where many other semigroup routines are to be found.

Numerical semigroups occur in various braches of mathematics, ranging from the study of singularities, number theory to coding theory. We hope this survey would be on interest to a larger audience, especially since there is a growing number of recent publications dealing with resolutions or Betti numbers for numerical semigroup rings. The results are scattered in various places, as witnessed by the list of references. We tried to be comprehensive, but the outcome is of course limited by our knowledge.

For most of the results we present we preferred not to include a full proof, but rather point to one, if available. A lot of examples are included, and it is here where we insisted on giving details, at places fixing some gaps in the literature.

\section{Algebraic WARM-UP}

In this section we give some basic algebraic facts about semigroup rings, their minimal resolution, and we recall some terminology.

Let $K$ be any field, $H$ a numerical semigroup minimally generated by $a_{1}, \ldots, a_{n}$ and $S=K\left[x_{1}, \ldots, x_{n}\right]$ the polynomial ring over $K$ in the indeterminates $x_{1}, \ldots, x_{n}$. On $S$ we consider the nonstandard grading given by the semigroup $H$ by letting $\operatorname{deg} x_{i}=a_{i}$ for $i=1, \ldots, n$. We denote $I_{H}$ the kernel of the $K$-algebra map $\phi$ : $S \rightarrow K[H]$ letting $\phi\left(x_{i}\right)=t^{a_{i}}$ for $i=1, \ldots, n$. The algebra $K[H] \cong S / I_{H}$ is a 
1-dimensional domain, hence by the Auslander-Buchsbaum formula ([14, Theorem 1.3.3]) we have that

$$
\text { proj } \operatorname{dim}_{S} K[H]=\text { height } I_{H}=n-1 .
$$

Let $f_{1}, \ldots, f_{r}$ be a minimal system of generators for $I_{H}$, which are homogeneous with respect to the grading on $S$ induced by the semigroup $H$. The relations among them are captured by the kernel of the $S$-linear map $\varphi_{1}: F_{1}=\bigoplus_{i=1}^{r} S e_{i} \rightarrow S$ letting $\varphi\left(e_{i}\right)=f_{i}$ for $i=1, \ldots, r$. To make this map homogeneous we assign $\operatorname{deg}\left(e_{i}\right)=\operatorname{deg}\left(f_{i}\right)$ for $i=1, \ldots, r$.

This process continues and we obtain a chain complex of free $S$-modules of finite rank

$$
\mathbb{F}: \quad 0 \rightarrow F_{n-1} \stackrel{\varphi_{n-1}}{\rightarrow} F_{n-2} \rightarrow \cdots \rightarrow F_{1} \stackrel{\varphi_{1}}{\rightarrow} F_{0}=S \rightarrow 0,
$$

which is exact in positive homological degrees, it has $H_{0}(\mathbb{F}) \cong S / I_{H}$, and the maps have the property that $\varphi_{i}\left(F_{i}\right) \subset \mathfrak{n} F_{i-1}$ for all $i=1, \ldots, n-1$. Here $\mathfrak{n}$ denotes the maximal homogeneous ideal of $S$. One says that $\mathbb{F}$ is a minimal free $S$-resolution of $S / I_{H}$. Such an $\mathbb{F}$ is unique (only) up to an isomorphism of chain complexes, hence we can define the $i^{\text {th }}$ Betti number of $K[H]$ as

$$
\beta_{i}(K[H])=\operatorname{rank} F_{i}=\operatorname{dim}_{K} \operatorname{Tor}_{i}^{S}(K[H], K) \text { for all } i,
$$

and this number does not depend on the chosen minimal free resolution of $K[H]$. The Betti sequence of $K[H]$ is the list $\left(\beta_{0}(K[H]), \beta_{1}(K[H]), \ldots\right)$. Clearly, $\beta_{0}(K[H])=1$ and $\beta_{1}(K[H])=\mu\left(I_{H}\right)$ the minimal number of generators for $I_{H}$.

Assume $G(H)=\left\{a_{1}, \ldots, a_{n}\right\}$ and $S=K\left[x_{1}, \ldots, x_{n}\right]$. Then $I_{H}$ is the binomial ideal

$$
I_{H}=\left(x^{u}-x^{v}: u, v \in \mathbb{N}^{n}, \sum_{i=1}^{n} u_{i} a_{i}=\sum_{i=1}^{n} v_{i} a_{i}\right),
$$

where for $u=\left(u_{1}, \ldots, u_{n}\right)$ we let $x^{u}=x_{1}^{u_{1}} \cdots x_{n}^{u_{n}}$. The toric ideal $I_{H}$ can be computed via elimination in the extended polynomial ring $S[t]$ :

$$
I_{H}=\left(x_{1}-t^{a_{1}}, \ldots, x_{n}-t^{a_{n}}\right) S[t] \cap S,
$$

or via saturation, as follows. Let $u_{(1)}, \ldots, u_{(n-1)}$ be a $\mathbb{Z}$-basis for the subgroup $\left\{\left(u_{1}, \ldots, u_{n}\right) \in \mathbb{Z}^{n}: \sum_{i=1}^{n} u_{i} a_{i}=0\right\}$. Then $I_{H}$ is the saturation

$$
I_{H}=I_{L}:\left(x_{1} \cdots x_{n}\right)^{\infty}
$$

of the lattice ideal

$$
I_{L}=\left(x^{u_{(i)}^{+}}-x^{u_{(i)}^{-}}: i=1, \ldots, n-1\right) .
$$

Here, for any vector $u$, by $u^{+}$and $u^{-}$we denote the unique vectors with nonnegative entries having disjoint support such that $u=u^{+}-u^{-}$. We refer to [70, Chapters 4, 12] for detailed proofs and further algorithms.

When $\operatorname{emb} \operatorname{dim}(H)=2, I_{H}$ is a principal ideal. If emb $\operatorname{dim}(H)=3$, Herzog [37] showed that $I_{H}$ can be generated by at most 3 binomials, see also [23]. For each $1 \leq i \leq 3$, we look at the smallest positive multiple $c_{i} a_{i}$ which is in the semigroup 
generated by the other two generators of $H$, and this gives a binomial generator for $I_{H}$. For instance, when $H=\langle 6,7,10\rangle$ we may write

$$
\begin{aligned}
& 4 \cdot 6=2 \cdot 7+1 \cdot 10, \\
& 4 \cdot 7=3 \cdot 6+1 \cdot 10, \\
& 2 \cdot 10=1 \cdot 6+2 \cdot 7,
\end{aligned}
$$

and this gives

$$
I_{H}=\left(x^{4}-y^{2} z, y^{4}-x^{3} z, z^{2}-x y^{2}\right) .
$$

In higher embedding dimension, it is more difficult in general to establish a system of generators for $I_{H}$ without using specialized software.

The maps in the resolution $\mathbb{F}$ are homogeneous with respect to the grading induced by the semigroup, and this is reflected in the decomposition of the Betti numbers as sum of their multigraded parts:

$$
\beta_{i}(K[H])=\sum_{\lambda \in H} \beta_{i, \lambda}(K[H]) .
$$

These summands can be expressed in terms of combinatorial and topological data. In [13] and in [15] for any $\lambda$ in $H$, the squarefree divisor complex $\Delta_{\lambda}$ is defined as the simplicial complex on the vertex set $\{1, \ldots, n\}$ where $\left\{i_{1}, \ldots, i_{r}\right\}$ is a face of $\Delta_{\lambda}$ if $\lambda-a_{i_{1}}-\cdots-a_{i_{r}} \in H$. Here is a way to make use of its reduced homology groups.

Theorem 2.1. (Bruns-Herzog [13, Proposition 1.1], Campillo-Marijuan [15, Theorem 1.2]) In the above notation,

$$
\beta_{i, \lambda}(K[H])=\operatorname{dim}_{K} \widetilde{H}_{i-1}\left(\Delta_{\lambda} ; K\right)
$$

for all $i>0$ and $\lambda$ in $H$.

The reduced homology modules of $\Delta_{\lambda}$ may depend on the characteristic of the field $K$, hence the same is true for the Betti numbers of $K[H]$. However, the number of connected components of $\Delta_{\lambda}$, which is given by $\operatorname{dim}_{K} H_{0}\left(\Delta_{\lambda} ; K\right)$ does not depend on $K$ and thus $\beta_{1}(K[H])=\mu\left(I_{H}\right)$ depends only on $H$. We refer to [13] for more results on this direction.

The tangent cone of $K[H]$ (or of $H$ ) is the associated graded ring of $K[H]$ with respect to the maximal ideal $\mathfrak{m}=\left(t^{h}: h \in H \backslash\{0\}\right) K[H]$, namely

$$
\operatorname{gr}_{\mathfrak{m}} K[H]=K[H] / \mathfrak{m} \oplus \mathfrak{m} / \mathfrak{m}^{2} \oplus \mathfrak{m}^{2} / \mathfrak{m}^{3} \oplus \cdots
$$

It is a standard graded $K$-algebra by letting $\mathfrak{m}^{i} / \mathfrak{m}^{i+1}$ be its homogeneous component of degree $i$. This grading is the one we shall further use for $\operatorname{gr}_{\mathfrak{m}} K[H]$, unless otherwise specified.

For any nonzero $f \in S$, its initial form $f^{*}$ is the homogeneous component (with respect to the standard grading) of smallest degree. For any ideal $I$ in $S$ we denote $I^{*}=\left(f^{*}: 0 \neq f \in I\right)$ the ideal of initial forms. If $f_{1}, \ldots, f_{r} \in I$ such that $I^{*}=\left(f_{1}^{*}, \ldots, f_{r}^{*}\right)$ one says that $\left\{f_{1}, \ldots, f_{r}\right\}$ is a standard basis of $I$. Moreover, in that situation the polynomials $f_{1}, \ldots, f_{r}$ generate $I$. 
The ideal $I^{*}$ is obtained from a set of generators $I=\left(f_{1}, \ldots, f_{r}\right)$ as follows. Let $F_{i}$ be the homogenization of $f_{i}$ with respect to a new variable $x_{0}$, for $i=1, \ldots, r$, and assume $G_{1}, \ldots, G_{s}$ is a Gröbner basis for the ideal $\left(F_{1}, \ldots, F_{r}\right) \subset S\left[x_{0}\right]$ with respect to a term order that refines the partial order by degree in $x_{0}$. If we set $g_{i}=G_{i}\left(1, x_{1}, \ldots, x_{n}\right)$ for $i=1, \ldots, s$ then $I_{H}^{*}=\left(g_{1}, \ldots, g_{s}\right)$, see [24, Proposition 15.28] or [26, Proposition 3.25] for a proof.

The relevance to us of this construction stems from the fact that $\mathrm{gr}_{\mathfrak{m}} K[H] \cong S / I_{H}^{*}$.

One can verify that for $H=\langle 6,7,10\rangle$ the three generators listed in (1) are a standard basis, hence $I_{H}^{*}=\left(y^{2} z, y^{4}-x^{3} z, z^{2}\right)$.

General deformation arguments (see [24]) prove that

$$
\beta_{i}(K[H]) \leq \beta_{i}\left(\operatorname{gr}_{\mathfrak{m}} K[H]\right) \text { for all } i,
$$

which shows that when searching for upper bounds for the Betti numbers of $K[H]$, we may refer to the Betti numbers of its tangent cone. In practice, the latter are easier to compute due to the standard grading on $I_{H}^{*}$. When $K[H]$ and $\operatorname{gr}_{\mathfrak{m}} K[H]$ have the same Betti sequence, one says that $K[H]$ (or $H$ ) is of homogeneous type, following the terminology of [40]. In [44, Theorem 3.17] the authors give a sufficient condition for $H$ to be of homogeneous type.

Both algebras $K[H]$ and $\operatorname{gr}_{\mathfrak{m}} K[H]$ have Krull dimension one, but while the former is a domain, the latter is not reduced and depth $\operatorname{gr}_{\mathfrak{m}} K[H] \leq 1$. By the AuslanderBuchsbaum formula,

$$
\operatorname{emb} \operatorname{dim}(H)-1 \leq \operatorname{proj} \operatorname{dim} \operatorname{gr}_{\mathfrak{m}} K[H] \leq \operatorname{emb} \operatorname{dim}(H) .
$$

The case $\operatorname{emb} \operatorname{dim}(H)-1=\operatorname{proj} \operatorname{dim} \operatorname{gr}_{\mathfrak{m}} K[H]$ is equivalent to depth $\operatorname{gr}_{\mathfrak{m}} K[H]=$ 1 (hence, by definition, $\operatorname{gr}_{\mathfrak{m}} K[H]$ is Cohen-Macaulay), i.e. there exists a regular element of positive degree in $\operatorname{gr}_{\mathfrak{m}} K[H]$. In our setting, this is equivalent to $\widehat{t^{\mathrm{m}(H)}}$ being regular on $\operatorname{gr}_{\mathfrak{m}} K[H]$.

Quite a bit of work (e.g. [46], [6], [3], [5], [38], [62], [31], [8], [41]) was directed towards finding criteria to test if $\operatorname{gr}_{\mathfrak{m}} K[H]$ is Cohen-Macaulay for an arbitrary numerical semigroup, partly motivated by the fact that in that situation the Hilbert function can be computed easier and it is non-decreasing.

The following hierarchy of rings is known: complete intersection $\Rightarrow$ Gorenstein $\Rightarrow$ Cohen-Macaulay. We explain what these conditions mean for our algebras of interest.

The algebra $K[H]$ or $\operatorname{gr}_{\mathfrak{m}} K[H]$ is a complete intersection (CI for short) if its defining ideal can be generated by the minimum number of polynomials allowed by Krull' s theorem, namely by height $I_{H}=$ height $I_{H}^{*}=\operatorname{emb} \operatorname{dim} H-1$ elements. The CI property for $K[H]$ and $\operatorname{gr}_{\mathfrak{m}} K[H]$ does not depend on the field $K$, but on some arithmetic conditions among the generators of $H$. Delorme [22] proved that $K[H]$ is CI if and only if the generators of $H$ can be obtained recursively via a process which is nowadays called gluing. D'Anna, Micale and Sammartano [19, Theorem 3.6] characterized the CI property for $\operatorname{gr}_{\mathfrak{m}} K[H]$ using the Apéry set of $H$.

One says that $K[H]$ or $\operatorname{gr}_{\mathfrak{m}} K[H]$ is a Gorenstein ring if it is Cohen-Macaulay and its type equals one. The Cohen-Macaulay condition always holds for $K[H]$. Kunz 
[49] showed that $K[H]$ is Gorenstein if and only if the semigroup $H$ is symmetric, i.e. for all $x \in \mathbb{Z}$ either $x \in H$ or $F(H)-x \in H$. The Gorenstein property for $\operatorname{gr}_{\mathfrak{m}} K[H]$ was characterized by Bryant in [12, Theorem 3.14]. One special feature of Gorenstein algebras is that their Betti sequence is symmetric, see [14, Theorem 3.3.7 (a), Corollary 3.3.9].

\section{The type of a numerical SEmigroup}

The Apéry set of the semigroup $H$ with respect to a nonzero integer $a$ in $H$ is

$$
\operatorname{Ap}(H, a)=\{x \in H: x-a \notin H\} .
$$

Clearly, its elements give different remainders modulo $a$ and $|\operatorname{Ap}(H, a)|=a$. The Apéry set of $H$ is defined as $\operatorname{Ap}(H, \mathrm{~m}(H))$.

The pseudo-Frobenius numbers of $H$ are the elements in

$$
P F(H)=\{x \in \mathbb{Z} \backslash H: x+h \in H \text { for all } h \in H, h>0\} .
$$

Fröberg, Gottlieb and Häggkvist in [28] define the type of $H$ by $\mathrm{t}(H)=|P F(H)|$.

In the following we explain why this purely arithmetic invariant equals the type of the semigroup ring $K[H]$, where $K$ is any field. We recall some algebraic terminology.

The type of a Cohen-Macaulay local ring $(R, \mathfrak{m})$ is $\mathrm{t}(R)=\operatorname{dim}_{K} \operatorname{Ext}_{R}^{d}(K, R)$, where $K=R / \mathfrak{m}$ and $d$ is the Krull dimension of $R$. Moreover, it $x_{1}, \ldots, x_{d} \subset \mathfrak{m}$ is an $R$-regular sequence then $\mathrm{t}(R)=\operatorname{dim}_{K} \operatorname{Hom}_{R}\left(K, R /\left(x_{1}, \ldots, x_{d}\right)\right)$, see [14, Lemma 1.2.19]. In case $R=A / I$ with $A$ a regular local ring and $I$ an ideal in $A$, then $\mathrm{t}(R)=\operatorname{dim}_{K} \operatorname{Tor}_{d}^{A}(K, R)$, see [2, Lemma 3.5]. This result means that $\mathrm{t}(R)$ is the rank of the last nonzero module in the minimal free resolution of $R$ over $A$.

The type of a Cohen-Macaulay ring $R$ is defined as the maximum of $\mathrm{t}\left(R_{\mathfrak{p}}\right)$, where $\mathfrak{p}$ ranges in the set of maximal ideals of $R$.

Theorem 3.1. Let $K$ be any field and $H$ be any numerical semigroup. Then $\mathrm{t}(H)=$ $\mathrm{t}(K[H])$.

Proof. The ring $R=K[H]$ is positively graded by setting $\operatorname{deg} t^{h}=h$ for all $h$ in $H$. $R$ has a unique maximal graded ideal $\mathfrak{m}$, hence by $[2$, Theorem pp. 75$]$ we get that $\mathrm{t}(K[H])=\mathrm{t}\left(K[H]_{\mathfrak{m}}\right)$. The ring map $K[H]_{\mathfrak{m}} \rightarrow K[|H|]$ is flat and its fiber is the field $K$, hence it follows from [14, Proposition 1.2.16.(b)] that $\mathrm{t}\left(K[H]_{\mathfrak{m}}\right)=\mathrm{t}(K[|H|])$.

Let $h \in H, h>0$. Since $t^{h}$ is a regular element on $K[|H|]$ we have that $\mathrm{t}(K[|H|])=\operatorname{dim}_{K} \operatorname{Hom}_{K[|H|]}\left(K, K[|H|] /\left(t^{h}\right)\right)$. It is an easy exercise to check that a $K$-basis for $\operatorname{Hom}_{K[|H|]}\left(K, K[|H|] /\left(t^{h}\right)\right)$ is given by the residue classes $\widehat{t^{x}}$ where $x$ ranges in the set

$$
B=\{x \in \operatorname{Ap}(H, h): x \neq h \text { and } x+g \in h+H \text { for all } g \in H \backslash 0\} .
$$

We also leave it to the reader to check the equality of sets $B=h+P F(H)$. Therefore,

$$
\mathrm{t}(H)=|P F(H)|=|B|=\mathrm{t}(K[|H|])=\mathrm{t}(K[H]),
$$

which finishes the proof.

From the arithmetic definition of the type of $H$ one gets the following inequalities. 
Theorem 3.2. (Fröberg, Gottlieb, Häggkvist [28]) Let H be a numerical semigroup. Then

(i) $\mathrm{t}(H)<\mathrm{m}(H)$;

(ii) if $\operatorname{emb} \operatorname{dim}(H) \leq 3$ then $\mathrm{t}(H) \leq 2$;

(iii) $(\mathrm{t}(H)+1) \cdot n(H) \geq F(H)+1$, where $n(H)$ is the number of elements in $H$ which are smaller that its Frobenius number $F(H)$.

By a result of Kunz [49] a semigroup is symmetric if and only if its type equals 1. When $H$ is pseudosymmetric, its type equals 2 , but the converse is not true. For instance $H=\langle 5,6,7\rangle$ has $P F(H)=\{8,9\}$.

In embedding dimension at least 4 there is no absolute upper bound on the type of the semigroup, as the following examples show; see also Section 8. Historically, Backelin was the first to produce 4-generated semigroups whose type is arbitrarily large, see the next example.

Example 3.3. (Backelin, [28, Example pp. 75]) Given the integers $n \geq 2$ and $r \geq 3 n+2$, set $s=r(3 n+2)+3$ and

$$
H=\langle s, s+3, s+3 n+1, s+3 n+2\rangle .
$$

It is proven in [28] that $\mathrm{t}(H) \geq 2 n+2$. However, it is wrongly claimed in [28] that $\mathrm{t}(H)=2 n+3$. Computations with Singular ([20]) and GAP ([30], [21]) indicate that both $K[H]$ and $\operatorname{gr}_{\mathfrak{m}} K[H]$ have the Betti sequence $(1,3 n+4,6 n+5,3 n+2)$.

For the next example the type was computed by Cavaliere and Niesi in [16]. They also show that for these semigroups the associated projective monomial curve is Cohen-Macaulay, which is not the case for Bresinsky's semigroups discussed in detail in Section 8.1.

Example 3.4. ([16, (3.4)]) For $a \geq 3$ let

$$
H_{a}=\left\langle a^{2}-a, a^{2}-a+1, a^{2}-1, a^{2}\right\rangle .
$$

Then $\mathrm{t}\left(H_{a}\right)=2 a-4$. Moreover, based on computations with Singular [20] we claim that the Betti sequences for $K\left[H_{a}\right]$ and $\operatorname{gr}_{\mathfrak{m}} K\left[H_{a}\right]$ are the same $(1,2 a-2,4 a-7,2 a-$ $4)$.

Combining Example 3.4 and Proposition 8.3 we see that any positive integer appears as the type of a 4-generated numerical semigroup. By the technique of gluing, such examples may be constructed for any embedding dimension larger than four, see Corollary 5.3.

When $H$ is generated by an (almost) arithmetic sequence, there are sharp bounds for the type in terms of the embedding dimension, see also Eq. (2).

Proposition 3.5. (i) (Tripathi, [73]) If $H$ is generated by an arithmetic sequence then $\mathrm{t}(H) \leq$ emb $\operatorname{dim}(H)-1$.

(ii) (García Marco, Ramírez Alfonsin, Rødseth, [32, Theorem 3.1]) If $H$ is generated by an almost arithmetic sequence then $\mathrm{t}(H) \leq 2(\operatorname{emb} \operatorname{dim}(H)-2)$. 
As noted before, symmetric semigroups have type 1. It is a natural question if for closely related classes of semigroups the type is at least bounded by (a linear function in) the embedding dimension.

Almost symmetric semigroups have been introduced by Barucci and Fröberg [7] as a class of semigroups close to the symmetric ones. The semigroup $H$ is almost symmetric if for every $x \in \mathbb{Z} \backslash H$ such that $F(H)-x \notin H$ we have $\{x, F(H)-x\} \subseteq$ $P F(H)$; see also [54] for equivalent characterizations.

Answering a question of Numata in [55], Moscariello [52] proves the following result, see also [43].

Theorem 3.6. ([52, Theorem 1]) If $H$ is almost symmetric and $\operatorname{emb} \operatorname{dim}(H)=4$ then $\mathrm{t}(H) \leq 3$, which is a sharp bound.

In embedding dimension larger than 4 there are examples of almost symmetric semigroups with $\mathrm{t}(H) \geq \operatorname{emb} \operatorname{dim}(H)$. Starting from such a semigroup, Strazzanti in [69, Remark 2.6.3, Example 2.6.4] constructs an almost symmetric semigroup $L$ of higher embedding dimension with $\mathrm{t}(L)-\operatorname{emb} \operatorname{dim}(L)>\mathrm{t}(H)-\operatorname{emb} \operatorname{dim}(H)$. This shows that there is no constant $c$ such that $\mathrm{t}(H) \leq \operatorname{emb} \operatorname{dim}(H)+c$ for every almost symmetric semigroup $H$.

Extending a question asked by Moscariello in [52] we ask the following.

Question 3.7. Is there any bound for the type and the rest of the Betti numbers for $K[H]$ in terms of emb $\operatorname{dim}(H)$ when $K[H]$ is almost/nearly Gorenstein?

\section{Shifted FAMilies of SEMigroups AND UPPER BOUNDS FOR THE NUMBER OF DEFINING EQUATIONS}

A recent idea used in the study of Betti numbers of semigroup rings was to examine their behaviour in families of semigroups. Firstly, for any sequence of nonnegative integers $\mathbf{a}: a_{1}<\cdots<a_{n}$ it will be convenient to denote in this section by $I(\mathbf{a})$ the kernel of the $K$-algebra homomorphism $\varphi: K\left[x_{1}, \ldots, x_{n}\right] \rightarrow K[t]$ letting $\varphi\left(x_{i}\right)=t^{a_{i}}$ for $i=1, \ldots, n$. For any integer $k$ we set $\mathbf{a}+k: a_{1}+k, \ldots, a_{n}+k$ and we call $\{\langle\mathbf{a}+k\rangle\}_{k \geq 0}$ the shifted family of semigroups associated to the sequence a. Herzog and Srinivasan conjectured that the Betti numbers for the semigroup rings in this shifted family are eventually periodic in $k$, for $k \gg 0$. After partial results in [33], [50] and [45], that conjecture was proved by $\mathrm{Vu}$ [74] in the following generality.

Theorem 4.1. (Vu, [74, Theorem 1.1]) Let $\mathbf{a}=a_{1}<\cdots<a_{n}$. There exists $k_{V}$ such that

$$
\beta_{i}(I(\mathbf{a}+k))=\beta_{i}\left(I\left(\mathbf{a}+k+\left(a_{n}-a_{1}\right)\right)\right)
$$

for all $i$ and all $k>k_{V}$.

We fix a and denote $H_{k}=\langle\mathbf{a}+k\rangle$. A key ingredient in Vu's proof is the following fact.

Proposition 4.2. ([74, Corollary 3.7]) For $k \gg 0$ the nonhomogeneous binomials in a minimal binomial system of generators for $I(\mathbf{a}+k)$ are of the form $x_{1}^{u_{1}} x_{2}^{u_{2}} \ldots x_{n-1}^{u_{n-1}}-$ $x_{2}^{v_{2}} \ldots x_{n-1}^{v_{n-1}} x_{n}^{v_{n}}$ with $u_{i} v_{i}=0$ for $i=2, \ldots, n-1, u_{1}, v_{n}>0$ and $\sum_{i=1}^{n-1} u_{i}>\sum_{i=2}^{n} v_{i}$. 
With the translation $k \mapsto k+\left(a_{n}-a_{1}\right)$, what changes in these binomials are the exponents of $x_{1}$ and $x_{n}$ which increase by a quantity that is also periodic with period $a_{n}-a_{1}$.

Proposition 4.2 was used by Herzog and Stamate ([41]) to derive that:

Theorem 4.3. ([41, Theorem 1.4]) For $k>k_{V}$ the ring $\operatorname{gr}_{\mathfrak{m}} K\left[H_{k}\right]$ is CohenMacaulay and it has the same Betti sequence as $K\left[H_{k}\right]$.

In [44, Corollary 6.5] Jafari and Zarzuela reprove this fact noticing that for $k \gg 0$ the semigroup $H_{k}$ is homogeneous, a concept they introduce.

As a corollary of these periodicity results, we see that in any of the families $\left\{K\left[H_{k}\right]\right\}_{k \geq 0}$ or $\left\{\operatorname{gr}_{\mathfrak{m}} K\left[H_{k}\right]\right\}_{k \geq 0}$ properties like Gorenstein or complete intersection occur either eventually with period $a_{n}-a_{1}$, or only for a finite set of shifts $k$.

The bound $k_{V}$ given in [74] is usually not optimal, and it involves the CastelnuovoMumford regularity of the ideal $J(\mathbf{a})$ of homogeneous polynomials in $I(\mathbf{a}+k)$ for some (and hence for all) $k$.

In case $n=3$, these statements may be sharpened. Note that by the results in Section 7.2, the periodicity for the Betti sequence of $K\left[H_{k}\right]$ now means that for $k \gg 0$ the semigroup $H_{k}$ is symmetric (actually CI) with period $a_{3}-a_{1}$. The principal (i.e. the smallest) period might be smaller, and it is determined in [68, Theorem 3.1] in terms of the sequence a. A smaller value than $k_{V}$ for the shift $k$ where periodicity occurs is provided in [68]. Also, in that paper exact formulas are conjectured for the thresholds from where the Betti numbers of $K\left[H_{k}\right]$, respectively of $\operatorname{gr}_{\mathfrak{m}} K\left[H_{k}\right]$ start changing periodically.

Another corollary of Theorems 4.1 and 4.3 is that if we bound the width of the semigroup $H$, the Betti numbers of $K[H]$ and $\operatorname{gr}_{\mathfrak{m}} K[H]$ are bounded, as well. We recall that the width of the numerical semigroup $H$ is the difference between the largest and the smallest minimal generator of $H$.

Two statements have been formulated in [41] regarding the number of generators for $I_{H}^{*}$ (hence also for $I_{H}$ ). Firstly, for a numerical semigroup $H$ minimally generated by $a_{1}<\cdots<a_{n}$ we define its interval completion to be the semigroup $\widetilde{H}$ generated by all the integers in the interval $\left[a_{1}, a_{n}\right]$.

Conjecture 4.4. ([41, Conjectures 2.1, 2.4]) For any numerical semigroup $H$ one has

(i) $\mu\left(I_{H}^{*}\right) \leq\left(\begin{array}{c}\operatorname{wd}(H)+1 \\ 2\end{array}\right)$,

(ii) $\mu\left(I_{H}^{*}\right) \leq \mu\left(I_{\widetilde{H}}^{*}\right)$.

If correct, the second point of the conjecture above would imply the first one. Also, since $\widetilde{H}$ is generated by an arithmetic sequence, we may use Eqs. (2) and obtain effective bounds for $\mu\left(I_{H}^{*}\right)$.

Betti numbers for intersections of toric ideals (and of their ideals of initial forms) of numerical semigroups in the same shifted family are considered in [17]. Given the sequence $\mathbf{a}$ as above, let

$$
\mathcal{I}_{\mathcal{A}}(\mathbf{a})=\bigcap_{k \in \mathcal{A}} I(\mathbf{a}+k) \text { and } \mathcal{J}_{\mathcal{A}}(\mathbf{a})=\bigcap_{k \in \mathcal{A}} I(\mathbf{a}+k)^{*} .
$$


It is conjectured in [17] that their Betti numbers are preserved under shifting $\mathcal{A} \mapsto$ $\mathcal{A}+\left(a_{n}-a_{1}\right)$, if $\min \mathcal{A} \gg 0$; see [17, Proposition 2.3] for some proved cases.

Conaway et al. in [18], and O'Neill and Pelayo in [57] study factorization invariants, respectively the Apéry sets in shifted semigroups $H_{k}$ for $k \gg 0$. As a byproduct they obtain the following.

Proposition 4.5. ([18, Theorems 3.4, 4.9], [57, Theorem 4.9]) For $k>\left(a_{n}-a_{1}\right)^{2}-a_{1}$

(i) the number of minimal generators for $I(\mathbf{a}+k)$ and the type of $H_{k}$ are periodic in $k$ with period $a_{n}-a_{1}$;

(ii) the conclusion of Proposition 4.2 holds.

With the same argument as in [41], Proposition 4.5(ii) implies that $\operatorname{gr}_{\mathfrak{m}} K\left[H_{k}\right]$ is Cohen-Macaulay and it has the same Betti sequence as $K\left[H_{k}\right]$ already for $k>$ $\left(a_{n}-a_{1}\right)^{2}-a_{1}$, improving Theorem 4.3 .

\section{BetTi NUMBERS FOR SIMPLE GLUINGS}

Having its roots in describing the structure of CI semigroups ([22], [75], [37]), the technique of gluing has been used to create interesting examples. We refer to Delorme's [22], Rosales' [63] and the monograph [64] for a more general definition.

For our purposes, we consider a special case. Let $L$ be a numerical semigroup minimally generated by $a_{1}<\cdots<a_{n}$, and $c>1$ and $d$ coprime integers such that $d \in L \backslash\left\{a_{1}, \ldots, a_{n}\right\}$. Then

$$
H=\langle c L, d\rangle=\left\langle c a_{1}, \ldots, c a_{n}, d\right\rangle
$$

is said (in [42]) to be obtained from $L$ by a simple gluing.

The order of $d$ in $L$ is defined as $\operatorname{ord}_{L}(d)=\max \left\{\sum_{i=1}^{n} \lambda_{i}: d=\sum_{i=1}^{n} \lambda_{i} a_{i}, \lambda_{i} \in \mathbb{N}\right\}$.

If we write $d=\lambda_{1} a_{1}+\cdots+\lambda_{n} a_{n}$ with $\lambda_{i}$ nonnegative integers and $\sum_{i=1}^{n} \lambda_{i}$ maximal, and we consider the gluing relation

$$
f=x_{n+1}^{c}-\prod_{i=1}^{n} x_{i}^{\lambda_{i}},
$$

then it is known (e.g. from the proof of Lemma 1 in [75], or [63, Theorem 1.4]) that

$$
I_{H}=\left(I_{L}, f\right) .
$$

The following lemma is the key step in describing the Betti numbers of $K[H]$ and $\operatorname{gr}_{\mathfrak{m}} K[H]$ in terms of the Betti numbers of $K[L]$ and $\operatorname{gr}_{\mathfrak{m}} K[L]$, respectively.

Lemma 5.1. ([42, Theorem 2.3, Lemma 2.6]) With notation as above, the following hold:

(i) $f$ is regular on $K\left[x_{1}, \ldots, x_{n+1}\right] / I_{L}$,

(ii) if $c \leq \operatorname{ord}_{L} d$, then $f^{*}$ is regular on $K\left[x_{1}, \ldots, x_{n+1}\right] / I_{L}^{*}$ and $I_{H}^{*}=\left(I_{L}^{*}, f^{*}\right)$.

Here is the main result of this section.

Theorem 5.2. Let $L$ be a numerical semigroup minimally generated by $a_{1}<\cdots<$ $a_{n}$, and $c>1$ and $d$ coprime integers with $d \in L \backslash\left\{a_{1}, \ldots, a_{n}\right\}$. Denote $H=\langle c L, d\rangle$. The following hold: 
(i) $\beta_{i}(K[H])=\beta_{i}(K[L])+\beta_{i-1}(K[L])$, for all $i>0$.

(ii) if $c \leq \operatorname{ord}_{L}(d)$, then $\beta_{i}\left(\operatorname{gr}_{\mathfrak{m}} K[H]\right)=\beta_{i}\left(\operatorname{gr}_{\mathfrak{m}} K[L]\right)+\beta_{i-1}\left(\operatorname{gr}_{\mathfrak{m}} K[L]\right)$, for all $i>0$.

Proof. Both statements are a consequence of Künneth's formula for the homology of the tensor product of two complexes, combined with Lemma 5.1. We give a detailed proof of part (i), the other one is proved similarly.

Denote $R=S\left[x_{n+1}\right]=K\left[x_{1}, \ldots, x_{n+1}\right]$. Let $\mathcal{L}$ be a minimal free $S$-resolution of $S / I_{L}$. Tensoring this over $S$ by $R$ we get the complex $\mathcal{P}$ which is a minimal free $R$-resolution of $R / I_{L} R$. We consider the complex $\mathcal{Q}: 0 \rightarrow R \stackrel{f}{\rightarrow} R \rightarrow 0$. Clearly $H_{i}(\mathcal{Q})=R /(f)$ if $i=0$, and 0 otherwise. Also, $\mathcal{Q}_{n}$ is free (and flat) for any $n$, and its image through the differential is either 0 , if $n \neq 0$, or $(f) R \cong R$, which is a free (and flat) $R$-module.

From Künneth's formula ([76, Theorem 3.6.3]), for any $n$ there exists a short exact sequence

$$
\left.0 \rightarrow \bigoplus_{i+j=n} H_{i}(\mathcal{P}) \otimes H_{j}(\mathcal{Q}) \rightarrow H_{n}(\mathcal{P} \otimes \mathcal{Q}) \rightarrow \bigoplus_{i+j=n-1} \operatorname{Tor}_{1}^{R}\left(H_{i}(\mathcal{P}), H_{j}(\mathcal{Q})\right)\right) \rightarrow 0
$$

For $n=0$ this gives $H_{0}(\mathcal{P} \otimes \mathcal{Q}) \cong H_{0}(\mathcal{P}) \otimes H_{0}(\mathcal{Q}) \cong R /\left(I_{L}, f\right)$, while for $n>1$ the previous exact sequence gives $H_{n}(\mathcal{P} \otimes \mathcal{Q})=0$.

Plugging in $n=1$ we get the exact sequence

$$
0 \rightarrow H_{1}(\mathcal{Q}) \rightarrow H_{1}(\mathcal{P} \otimes \mathcal{Q}) \rightarrow \operatorname{Tor}_{1}^{R}\left(H_{0}(\mathcal{P}), H_{0}(\mathcal{Q})\right) \rightarrow 0
$$

hence $H_{1}(\mathcal{P} \otimes \mathcal{Q}) \cong \operatorname{Tor}_{1}^{R}\left(R / I_{L} R, R /(f)\right)$. Note that by Lemma 5.1, $f$ is regular on $R$ and on $R / I_{L} R$, hence by [14, Proposition 1.1.5], the complex obtained by tensoring with $R /(f)$ the free resolution $\mathcal{P}$ of $R / I_{L} R$, is again exact. Therefore, $\operatorname{Tor}_{1}^{R}\left(R / I_{L} R, R /(f)\right)=0$ and $\mathcal{P} \otimes \mathcal{Q}$ is exact and a minimal free resolution of $R /\left(I_{L}, f\right)$.

Clearly, for $n>0$ one has $(\mathcal{P} \otimes \mathcal{Q})_{n}=\left(P_{n} \otimes Q_{0}\right) \oplus\left(P_{n-1} \otimes Q_{1}\right) \cong P_{n} \oplus P_{n-1}$. This shows that $\beta_{i}(K[H])=\beta_{i}(K[L])+\beta_{i-1}(K[L])$ for all $i>0$.

An immediate consequence of part (i) is the following result of Fröberg, Gottlieb and Häggkvist [28].

Corollary 5.3. ([28, Proposition 8]) If $H=\langle c L, d\rangle$ is a simple gluing, then $K[H]$ and $K[L]$, and also $H$ and $L$ have the same type.

Proof. Denoting $n=\operatorname{emb} \operatorname{dim}(L)$, since $K[H]$ is a Cohen-Macaulay $S$-module of dimension 1, by Theorem 5.2(i) the type of $K[H]$ is $\beta_{n}(K[H])=\beta_{n}(K[L])+$ $\beta_{n-1}(K[L])=\beta_{n-1}(K[L])$, hence $K[H]$ and $K[L]$ have the same type. The rest follows from Theorem 3.1.

Corollary 5.4. If $H=\langle c L, d\rangle$ is a simple gluing and $c \leq \operatorname{ord}_{L}(d)$, then $\operatorname{gr}_{\mathfrak{m}} K[H]$ is Cohen-Macaulay or Gorenstein if and only if $\operatorname{gr}_{\mathfrak{m}} K[L]$ is Cohen-Macaulay, respectively Gorenstein.

Proof. Let $n=\mathrm{emb} \operatorname{dim}(L)$. By the Auslander-Buchsbaum formula [14, Theorem 1.3.3], $\operatorname{gr}_{\mathfrak{m}} K[H]$ and $\operatorname{gr}_{\mathfrak{m}} K[L]$ are Cohen-Macaulay if and only if $\beta_{n}\left(\operatorname{gr}_{\mathfrak{m}} K[H]\right)=0$, 
respectively $\beta_{n+1}\left(\operatorname{gr}_{\mathfrak{m}} K[L]\right)=0$. The conclusion follows from Theorem 5.2(ii) and Corollary 5.3.

Remark 5.5. The condition $c \leq \operatorname{ord}_{L}(d)$ in Corollary 5.4 can not be removed. Indeed, for $h>1$ let $L=\langle 3 h, 3 h+1,6 h+3\rangle$ and $H=\left\langle\left(h^{2}+1\right) L, 3 h^{2}\right\rangle$ from Example 9.4. Clearly, $\operatorname{ord}_{L}\left(3 h^{2}\right)=h<h^{2}+1$. As noted in Examples 7.1 and 9.4 respectively, the tangent cone $\operatorname{gr}_{\mathfrak{m}} K[L]$ is not Cohen-Macaulay, however $\operatorname{gr}_{\mathfrak{m}} K[H]$ is Cohen-Macaulay.

Remark 5.6. A simple gluing $H=\langle c L, d\rangle$ with $c \leq \operatorname{ord}_{L}(d)$ is called a nice extension in [4]. This concept is generalized in [5, Definition 2.1] by Arslan, Mete and Şahin to nice gluings of arbitrary numerical semigroups. In [5, Theorem 2.6] the authors show that a nice gluing of two semigroups $H_{1}$ and $H_{2}$ with Cohen-Macaulay tangent cones, also has a Cohen-Macaulay tangent cone. This gives another proof for the Corollary 5.4 above. Şahin [71] studies nice extensions for arbitrary toric varieties, and he formulates the results in this section in that generality.

The Betti numbers of $K[H]$ when $H$ is a numerical semigroup obtained by gluing have also been considered in [34] and [56].

\section{SOME EXAMPLES}

For some classes of numerical semigroups the resolution of $K[H]$ and of $\operatorname{gr}_{\mathfrak{m}} K[H]$ is known.

6.1. Complete intersections. When $K[H]$ is a complete intersection ring, its defining ideal is generated by $a=\operatorname{emb} \operatorname{dim}(H)-1$ binomials which are a regular sequence in $S$, hence the Koszul complex on these binomials provides a minimal $S$ free resolution of $S / I_{H}$, see [14, Corollary 1.6.14]. Consequently, the Betti sequence for $K[H]$ is

$$
\left(1,\left(\begin{array}{l}
a \\
1
\end{array}\right),\left(\begin{array}{l}
a \\
2
\end{array}\right), \ldots,\left(\begin{array}{l}
a \\
a
\end{array}\right)\right) .
$$

The numerical semigroups $H$ for which $K[H]$ is CI have been characterized combinatorially by Delorme [22] in terms of gluings, see also Section 5. For instance, when $H$ is generated by a geometric sequence, the rings $\operatorname{gr}_{\mathfrak{m}} K[H]$ and $K[H]$ are CI, see [42, Proposition 3.4].

6.2. Arithmetic sequences. When $H=\langle a, a+d, \ldots, a+(n-1) d\rangle$ is generated by an arithmetic sequence with $\operatorname{gcd}(a, d)=1, n \leq a$, Gimenez, Sengupta and Srinivasan in [33] show that if we denote by $b$ the unique integer such that $a \equiv b \bmod (n-1)$ and $1 \leq b \leq n-1$, then

$$
\beta_{i}(K[H])=i\left(\begin{array}{c}
n-1 \\
i+1
\end{array}\right)+ \begin{cases}(n-b-i+1)\left(\begin{array}{c}
n-1 \\
i-1
\end{array}\right) & \text { if } 1 \leq i \leq n-b \\
(i-n+b)\left(\begin{array}{c}
n-1 \\
i
\end{array}\right) & \text { if } n-b<i \leq n-1\end{cases}
$$

see also the preprint [58] of Oneto and Tamone for an independent, yet similar approach.

The same values for the Betti numbers of $\operatorname{gr}_{\mathfrak{m}} K[H]$ had been obtained by Sharifan and Zaare-Nahandi in [65], and the equality $\beta_{i}(K[H])=\beta_{i}\left(\operatorname{gr}_{\mathfrak{m}} K[H]\right)$, for all $i$, was 
noted by them in [66]. Independently, in [41] using just the relations defining $K[H]$, Herzog and the author also showed that $\operatorname{gr}_{\mathfrak{m}} K[H]$ has the same Betti numbers as $K[H]$.

Formulas similar to (2) hold when $H$ is generated by a generalized arithmetic sequence, i.e. $H=\langle a, h a+d, h a+2 d, \ldots, h a+(n-1) d\rangle$ for some positive integers $h, d$ with $\operatorname{gcd}(a, d)=1$. Details, and also the explicit minimal free resolution of $K[H]$ may be found in [65], [33] and [59].

\section{Embedding dimension at most 3}

7.1. The 2-generated case. If $H=\left\langle a_{1}, a_{2}\right\rangle$ with $a_{1}<a_{2}$ and $\operatorname{gcd}\left(a_{1}, a_{2}\right)=1$, then $K[H] \cong K[x, y] /\left(x^{a_{2}}-y^{a_{1}}\right)$ and $\operatorname{gr}_{\mathfrak{m}} K[H] \cong K[x, y] /\left(y^{a_{1}}\right)$, hence both algebras have the Betti sequence $(1,1)$.

7.2. The 3-generated case. If emb $\operatorname{dim}(H)=3$, by Herzog's work in [37] one has that $\mu\left(I_{H}\right) \leq 3$, hence the possible Betti sequences of $K[H]$ are $(1,2,1)$ if it is CI, or $(1,3,2)$ if it is not. Moreover, Herzog [38] and Robbiano-Valla [62] show that $\operatorname{gr}_{\mathfrak{m}} K[H]$ is Cohen-Macaulay if and only if $\mu\left(I_{H}^{*}\right) \leq 3$, and therefore, under such an extra hypothesis the possible Betti sequences of $\operatorname{gr}_{\mathfrak{m}} K[H]$ are again $(1,3,2)$ and $(1,2,1)$.

On the other hand, there are examples of Shibuta ([35, Example 5.5]) of 3generated semigroups $H$ where $\mu\left(I_{H}^{*}\right)$ is arbitrarily large. Extending that family, one has the following

Example 7.1. ([41, Section 3.3]) For $a>3$ and

$$
H_{a}=\langle a, a+1,2 a+3\rangle
$$

one obtains that $\mu\left(I_{H_{a}}^{*}\right)=\left\lfloor\frac{a-1}{3}\right\rfloor+3$, by explicitly computing the ideal of initial forms

$$
I_{H_{a}}^{*}=\left(x z, z^{k+1}\right)+y^{\varepsilon} \cdot\left(y^{3}, z\right)^{k} .
$$

Here we wrote $a=3 k+\varepsilon$ with $k=\left\lfloor\frac{a-1}{3}\right\rfloor$ and $1 \leq \varepsilon \leq 3$. Numerical experiments with Singular [20] indicate that, in this notation, the whole Betti sequence of $\operatorname{gr}_{\mathfrak{m}} K\left[H_{a}\right]$ is $(1, k+3,2 k+2, k)$.

\section{LARGe Betti numbers in EMBEDding Dimension 4}

When emb $\operatorname{dim}(H) \geq 4$, there is no upper bound depending on emb $\operatorname{dim}(H)$ alone for the number of defining equations and the rest of the Betti numbers of $K[H]$, as the following examples show.

8.1. Bresinsky semigroups. For $h \geq 2$, Bresinsky [11] considered the numerical semigroup

$$
B_{h}=\langle(2 h-1) 2 h,(2 h-1)(2 h+1), 2 h(2 h+1), 2 h(2 h+1)+2 h-1\rangle .
$$

and he showed that the number of defining equations for $K\left[B_{h}\right]$ is at least $2 h$. We now compute its whole Betti sequence. 
Theorem 8.1. With notation as above, both algebras $K\left[B_{h}\right]$ and $\operatorname{gr}_{\mathfrak{m}} K\left[B_{h}\right]$ have the Betti sequence

$$
(1,4 h, 8 h-4,4 h-3) .
$$

Proof. We fix an integer $h \geq 2$ and for brevity we denote $I=I_{B_{h}} \subset S=K[x, y, z, t]$. Based on computations started in [11], it is proven in [41, Section 3.3] that

$\{x t-y z\} \cup\left\{z^{i-1} t^{2 h-i}-y^{2 h-i} x^{i+1}: 1 \leq i \leq 2 h\right\} \cup\left\{x^{2 h+1-j} z^{j}-y^{2 h-j} t^{j}: 0 \leq j \leq 2 h-2\right\}$

is a minimal generating set and a minimal standard basis of $I$, and that $\operatorname{gr}_{\mathfrak{m}} K\left[B_{h}\right]$ is Cohen-Macaulay.

We let $\bar{S}=K[y, z, t], \bar{I}$ be the canonical projection of $I \subset S$ onto $\bar{S}$, and similarly for $\bar{I}^{*}$. It is immediate to check that

$$
\bar{I}=\bar{I}^{*}=(y z)+\left(t^{2 h-1}, z t^{2 h-2}, \ldots, z^{2 h-1}\right)+\left(y^{2} t^{2 h-2}, y^{3} t^{2 h-3}, \ldots, y^{2 h-1} t, y^{2 h}\right) .
$$

Clearly $x$ is regular on $S / I$ which is a domain, but also on $S / I^{*}$ since $\operatorname{gr}_{\mathfrak{m}} K\left[B_{h}\right]$ is Cohen-Macaulay, as noted above. Therefore, the Betti numbers for $S / I, S / I^{*}$, and $\bar{S} / \bar{I}$ coincide. The conclusion follows from the next lemma.

Lemma 8.2. Consider the ideal $J \subset \bar{S}=K[y, z, t]$ defined as

$$
J=(y z)+\left(t^{h}, z t^{h-1}, \ldots, z^{h}\right)+\left(y^{2} t^{h-1}, y^{3} t^{h-2}, \ldots, y^{h+1}\right) .
$$

The Betti sequence of $\bar{S} / J$ is $(1,2 h+2,4 h, 2 h-1)$.

Proof. Clearly $\beta_{0}(\bar{S} / J)=1$ and $\beta_{1}(\bar{S} / J)=\mu(J)=2 h+2$.

Since $\bar{S} / J$ has finite length, it is Cohen-Macaulay. Hence its projective dimension as an $\bar{S}$-module equals 3 and its last nonzero Betti number satisfies $\beta_{3}(\bar{S} / J)=$ $\operatorname{dim}_{K} \operatorname{Soc}(\bar{S} / J)$. Note that

$$
\operatorname{Soc}(\bar{S} / J)=\{\widehat{f} \in \bar{S} / J:(y, z, t) \cdot \widehat{f}=\hat{0}\}
$$

has a $K$-basis consisting of the residue classes of the monomials $m \notin J$ such that $(y, z, t) \cdot m \subset J$.

Let $m=y^{a} z^{b} t^{c}$ be such a monomial. Clearly $a b=0$.

If $a=0$, then $b>0$, otherwise $m=y^{c} \notin J$ implies $c \leq h$, and together with $y m=y t^{c} \in J$ we get a contradiction. Moreover, $m=z^{b} t^{c} \notin J$ yields $b+c \leq h-1$. Since $z m \in J$, one gets that $b+c+1 \geq h$, hence $b+c=h-1$ and $m \in \mathcal{S}_{1}=$ $\left\{z t^{h-2}, z^{2} t^{h-3}, \ldots, z^{h-1}\right\}$.

If $a>0$, then $b=0$. Since $m=y^{a} t^{c} \notin J$ we get $c<h$. Also, $y m=y^{a+1} t^{c} \in J$ implies $a+1+c \geq h+1$, i.e. $a+c \geq h$. From $t m=y^{a} t^{c+1} \in J$ we get that either $a=1$ and $c+1=h$, or $a \geq 2$ and $a+c+1=h+1$. We obtain that $m \in \mathcal{S}_{2}=\left\{y t^{h-1}, y^{2} t^{h-2}, \ldots, y^{h}\right\}$. It is easy to check that $\widehat{m} \in \operatorname{Soc}(\bar{S} / J)$ for all $m \in \mathcal{S}_{1} \cup \mathcal{S}_{2}$, therefore $\beta_{3}(\bar{S})=2 h-1$.

From the relation $\sum_{i} \beta_{i}(\bar{S} / J)=0$ we see that $\beta_{2}(\bar{S})=4 h$, and this finishes the computation of the Betti sequence. 
8.2. Arslan semigroups. In [3, Proposition 3.2] Arslan shows that for the family of semigroups

$$
A_{h}=\left\langle h(h+1), h(h+1)+1,(h+1)^{2},(h+1)^{2}+1\right\rangle, \text { where } h \geq 2,
$$

the defining ideal of $K\left[A_{h}\right]$ is

$$
\begin{aligned}
I_{A_{h}}=\left(x^{h-i} z^{i+1}-y^{h-i+1} t^{i}: 0 \leq\right. & i<h)+ \\
& \left(z^{i} t^{h-i}-x^{i+1} y^{h-i}: 0 \leq i \leq h\right)+(x t-y z) .
\end{aligned}
$$

and later on he proves that $\operatorname{gr}_{\mathfrak{m}} K\left[A_{h}\right]$ is Cohen-Macaulay via some considerations involving Gröbner bases.

With notation as before, going modulo $x$ we obtain

$$
\bar{I}_{A_{h}}=\left(y^{h-i+1} t^{i}: 0 \leq i<h\right)+\left(z^{i} t^{h-i}: 0 \leq i \leq h\right)+(y z) .
$$

This is a monomial ideal whose generators, naturally forming a standard basis, may be clearly lifted to binomials in $I_{A_{h}}$ with the same initial degree. According to Herzog's criterion [38, Theorem 1], as formulated in [41, Lemma 1.2], we get that the generators in (5) for $I_{A_{h}}$ form a standard basis as well, and that $x$ is regular on $\operatorname{gr}_{\mathfrak{m}} K\left[A_{h}\right]$. Hence we reobtain Arslan's result in [3, Proposition 3.4]:

$$
I_{A_{h}}^{*}=\left(x^{h-i} z^{i+1}-y^{h-i+1} t^{i}: 0 \leq i<h\right)+\left(z^{i} t^{h-i}: 0 \leq i \leq h\right)+(x t-y z) .
$$

Modulo $x$, the latter ideal is the same as $\bar{I}_{A_{h}}$ and it coincides with the ideal $J$ in (3). Using Lemma 8.2, we derive the next result.

Proposition 8.3. Let $h \geq 2$ and $A_{h}$ be the Arslan semigroup defined in (4). The semigroup ring $K\left[A_{h}\right]$ and its tangent cone $\operatorname{gr}_{\mathfrak{m}} K\left[A_{h}\right]$ have the same Betti sequence $(1,2 h+2,4 h, 2 h-1)$.

These computations show that in the class of 4-generated numerical semigroups, even among those with Cohen-Macaulay tangent cone, the Betti numbers of $K[H]$ may be arbitrarily large. Using the gluing construction described in Section 5, we can exhibit examples of semigroups with arbitrarily large Betti numbers in any higher embedding dimension.

\section{Embedding Dimension 4, CONTINUED}

9.1. AA-sequences. A sequence of integers is called an almost arithmetic (AA) sequence if it consists of an arithmetic sequence and of one more element. Any 3 -generated semigroup is generated by an AA-sequence.

Kumar Roy, Sengupta and Tripathi [48] described the minimal resolution of $K[H]$ when $H$ is minimally generated by an AA-sequence with 4 elements. Similar to the results in Section 6.2, they obtain that only the following eight Betti sequences are possible: $(1,3,3,1),(1,4,5,2),(1,4,6,3),(1,5,5,1),(1,5,6,2),(1,5,7,3),(1,6,8,3)$, $(1,6,9,4)$. 
9.2. Symmetric semigroups. Assume $H$ is a 4-generated symmetric semigroup. If $H$ is CI, the Betti sequence of $K[H]$ is $(1,3,3,1)$, as seen in Section 6.1.

When $H$ is not CI, Bresinsky [10] described its generators and he explicitly computed the defining relations for $K[H]$. Based on that parametrization, Barucci, Fröberg and Şahin [9] described the minimal free resolution of $K[H]$. The Betti sequence is always $(1,5,5,1)$ in that case.

Remark 9.1. Micale and Olteanu [51] notice that in embedding dimension at least five, more than one Betti sequence is possible for $K[H]$ when $H$ is symmetric and not CI. Indeed, if $a$ and $d$ are coprime positive integers such that $a \equiv 2 \bmod (4)$, letting $H=\langle a, a+d, a+2 d, a+3 d, a+4 d\rangle$, it follows from Eq. (2) that the Betti sequence of $K[H]$ is $(1,9,16,9,1)$.

On the other hand, the semigroup $H=\langle 12,20,28,30,35\rangle$ is symmetric, not CI and the Betti sequence of $K[H]$ is $(1,6,10,6,1)$. A large class of symmetric semigroups with this Betti sequence is provided by [51, Proposition 5.1].

When $H$ is 4-generated, symmetric and not CI, it is a natural question to find the equations of the tangent cone, since we know the five equations defining $K[H]$. Despite the effort (see [46], [4]), explicit formulas are not available in all cases.

Arslan, Katsabekis and Nalbandiyan [6] gave necessary and sufficient conditions for a 4-generated symmetric and not CI semigroup to have a Cohen-Macaulay tangent cone, in terms of Bresinsky's parametrization; see also [46, Theorem 2.4] for a more compact formulation of their result.

Recently, under these hypotheses for $H$, building on the results in [6], Katsabekis [46] shows that in several cases when $\operatorname{gr}_{\mathfrak{m}} K[H]$ is Cohen-Macaulay, then $\mu\left(I_{H}^{*}\right) \in$ $\{5,6\}$ by explicitly finding a standard basis for $I_{H}$. It is likely that this program can be completed to find the possible Betti sequences of the tangent cone, at least in those several cases. We ask if that is a finite list or not. We also wonder if $\mu\left(I_{H}^{*}\right)$ can be determined in all the cases when $\operatorname{gr}_{\mathfrak{m}} K[H]$ is Cohen-Macaulay, compare with Example 9.4.

Here are some examples.

Example 9.2. We used Singular [20] to compute the Betti sequences of $\operatorname{gr}_{\mathfrak{m}} K[H]$ for some 4-generated symmetric and non-CI semigroups $H$ given in [6, Examples $3.14,3.21,3.28,4.3]$ :

(a) for $H$ any of $\langle 1199,2051,2352,3032\rangle,\langle 627,1546,1662,3377\rangle$, or $\langle 813,1032,1240,1835\rangle$ the tangent cone $\operatorname{gr}_{\mathfrak{m}} K[H]$ is Cohen-Macaulay and its Betti sequence is $(1,6,8,3)$;

(b) for $H=\left\langle 2 m+1,2 m+3,2 m^{2}+m-2,2 m^{2}+m-1\right\rangle$ with $m \geq 4$, the ideal $I_{H}^{*}$ is explicitly computed and one gets that $\operatorname{gr}_{\mathfrak{m}} K[H]$ is not Cohen-Macaulay, and its Betti sequence is $(1,8,14,9,2)$.

Example 9.3. Arslan and Mete (see [4, Example 2.1]) show that for $m \geq 2$ the semigroup

$$
H_{m}=\left\langle m^{3}+m^{2}-m, m^{3}+2 m^{2}+m-1, m^{3}+3 m^{2}+2 m-2, m^{3}+4 m^{2}+3 m-2\right\rangle
$$


is symmetric and not CI, and that $\operatorname{gr}_{\mathfrak{m}} K\left[H_{m}\right]$ is Cohen-Macaulay by explicitly computing

$$
I_{H_{m}}^{*}=\left(z t^{m-1}, y^{m+2}, z^{m}, t^{m}, y^{m} t^{m-1}\right) .
$$

Denoting $J=I_{H_{m}}^{*} \bmod x$, it is easy to check that $\left\{y^{m-1} z^{m-1} t^{m-2}, y^{m-1} t^{m-1}\right\}$ is a $K$-basis for $\operatorname{Soc}(\bar{S} / J)$, hence arguing as in the proof of Lemma 8.2 we get that $\operatorname{gr}_{\mathfrak{m}} K\left[H_{m}\right]$ has the Betti sequence $(1,5,6,2)$.

We next consider the possible number of equations for $\operatorname{gr}_{\mathfrak{m}} K[H]$ when $H$ is CI and $\operatorname{emb} \operatorname{dim}(H)=4$.

Recall that in embedding dimension $3, \operatorname{gr}_{\mathfrak{m}} K[H]$ is $\mathrm{CM}$ if and only if $\mu\left(I_{H}^{*}\right) \leq$ 3. Working to extend this result, Shibuta [67, Theorem 3.1] shows that if $H$ is a 4-generated CI numerical semigroup and $\mu\left(I_{H}^{*}\right) \leq 4$, then $\operatorname{gr}_{\mathfrak{m}} K[H]$ is CohenMacaulay. The paper [67] is also the source of the following example.

Example 9.4. (Shibuta, [67, Proposition 3.14]) For $h \geq 2$ the semigroup

$$
H_{h}=\left\langle 3 h^{2}, 3 h\left(h^{2}+1\right),(3 h+1)\left(h^{2}+1\right),(6 h+3)\left(h^{2}+1\right)\right\rangle
$$

is CI with $I_{H_{h}}=\left(y^{h}-x^{h^{2}+1}, y t-z^{3}, t^{h}-x^{2\left(h^{2}+1\right)} y\right) \subset S=K[x, y, z, t]$ and $I_{H_{h}}^{*}=$ $(y t)+\left(z^{3}, y\right)^{h}+\left(z^{3}, t\right)^{h}$.

Clearly $x$ is regular in $S / I_{H_{h}}^{*}$ and $\operatorname{gr}_{\mathfrak{m}} K\left[H_{h}\right]$ is Cohen-Macaulay. We shall find its Betti numbers as in the proof of Lemma 8.2. Letting $J=I_{H_{h}}^{*} \bmod x$ and $\bar{S}=$ $K[y, z, t]$, it is easy to check that

$$
\left\{y^{h-1} z^{3 i-1}: 1 \leq i \leq h\right\} \cup\left\{z^{3 i-1} t: 1 \leq i \leq h-1\right\}
$$

is a $K$-basis for $\operatorname{Soc}(\bar{S} / J)$. Therefore the Betti sequences for $\operatorname{gr}_{\mathfrak{m}} K\left[H_{h}\right]$ and for $\bar{S} / J$ are $(1,2 h+2,4 h, 2 h-2)$.

Example 9.5. (Katsabekis, [46, Example 3.6]) For $m \geq 1$ the semigroup

$$
H_{m}=\left\langle 8 m^{2}+6,8 m^{2}+10,12 m^{2}+15,20 m^{2}+15\right\rangle
$$

is CI, with $I_{H_{m}}=\left(x^{5}-t^{2}, y^{3}-z^{2}, x^{2 m^{2}} t-y^{2 m^{2}} z\right)$. Using the methods described in Section 2 one gets that $I_{H_{m}}^{*}=\left(z^{2}, t^{2}, x^{2 m^{2}} t-y^{2 m^{2}} z, y^{2 m^{2}+3} t, y^{4 m^{2}+3}\right)$, and the tangent cone is not Cohen-Macaulay. Testing with Singular ([20]) for small values of $m$ we always get the Betti sequence $(1,5,9,6,1)$ for $\operatorname{gr}_{\mathfrak{m}} K\left[H_{m}\right]$.

Starting with a 3 -generated CI semigroup $H$ whose tangent cone has large Betti numbers, by a simple gluing (see Section 5) we can obtain a 4-generated CI whose tangent cone has large Betti numbers, as well.

Example 9.6. For $m \geq 2$, the semigroup $H_{m}=\langle 3 m, 3 m+1,6 m+3\rangle$ in Example 7.1 is $\mathrm{CI}$ and the Betti sequence for $\operatorname{gr}_{\mathfrak{m}} K\left[H_{m}\right]$ is $(1, m+2,2 m, m-1)$. Letting

$$
L_{m}=\left\langle 2 H_{m}, 6 m+1\right\rangle=\langle 6 m, 6 m+2,12 m+6,6 m+1\rangle,
$$

we have that $L_{m}$ is CI. By Theorem 5.2, the tangent cone $\operatorname{gr}_{\mathfrak{m}} K\left[L_{m}\right]$, which is not Cohen-Macaulay, has the Betti sequence $(1, m+3,3 m+2,3 m-1, m-1)$.

Remark 9.7. Following Examples 9.4 and 9.6, we expect that arbitrarily large Betti numbers may be obtained for $\operatorname{gr}_{\mathfrak{m}} K[H]$ also when $H$ is 4-generated, symmetric and not CI. 
9.3. Pseudosymmetric semigroups. A semigroup $H$ is called pseudosymmetric if $F(H)$ is even and $P F(H)=\{F(H) / 2, F(H)\}$. Komeda [47] characterized the generators of any 4-generated pseudosymmetric numerical semigroup $H$ and he found the defining relations of $K[H]$. Based on that, Barucci, Fröberg and Şahin [9] described the minimal free resolution of $K[H]$. Consequently, its Betti sequence is $(1,5,6,2)$.

The defining equations or the Betti numbers of the tangent cone are not known for this class of semigroups. Şahin and Şahin ([72]) describe the Cohen-Macaulay property of $\operatorname{gr}_{\mathfrak{m}} K[H]$ in terms of Komeda's parametrization.

9.4. Further extensions. Assume $H$ is a 4-generated semigroup which is not CI. Eto [27] describes the almost symmetric such semigroups: their generators, the defining equations of $K[H]$ and also the minimal resolution of $K[H]$. When the type of $H$ is $2, H$ is pseudosymmetric and this was discussed above. Moscariello [52] had proven that otherwise $H$ must have type equal to 3, and Eto shows that the possible Betti sequences are $(1,6,8,3)$ and $(1,7,9,3)$. This completes some very partial results in [55].

The class of nearly Gorenstein semigroups has been recently introduced in [39] and it contains the almost symmetric ones.

Problem 9.8. Find a parametrization of the 4-generated nearly Gorenstein semigroups and describe the minimal resolution of their semigroup ring.

Problem 9.9. Describe the Betti numbers and the minimal resolution of $\operatorname{gr}_{\mathfrak{m}} K[H]$ when $H$ is a 4-generated semigroup which is (almost) symmetric or nearly Gorenstein.

Acknowledgement. We gratefully acknowledge the use of the Singular [20] software and of the numericalsgps package [21] in GAP [30] for the development of this paper. The author was supported by a fellowship at the Research Institute of the University of Bucharest (ICUB).

We thank Ignacio García-Marco for sending corrections to an initial version of this paper, to Francesco Strazzanti for useful pointers to the literature and to an anonymous referee for suggestions that improved the presentation. A great moral debt is owed to Jürgen Herzog since our joint projects served as an introduction to the topic of this survey and also as a motivation to write it.

\section{REFERENCES}

[1] J. Abbott, A. M. Bigatti, G. Lagorio, CoCoA-5: a system for doing Computations in Commutative Algebra. http://cocoa.dima.unige.it.

[2] Y. Aoyama, S. Goto, On the type of graded Cohen-Macaulay rings, J. Math. Kyoto Univ. 15 (1975), 19-23.

[3] F. Arslan, Cohen-Macaulayness of tangent cones, Proc. Amer. Math. Soc. 128 no. 8 (1999), $2243-2251$.

[4] F. Arslan, P. Mete, Hilbert functions of Gorenstein monomial curves, Proc. Amer. Math. Soc. 135 (2007), 1993-2002. 
[5] F. Arslan, P. Mete, M. Şahin, Gluing and Hilbert functions of monomial curves, Proc. Amer. Math. Soc. 137 (2009), 2225-2232.

[6] F. Arslan, A. Katsabekis, M. Nalbandiyan, On the Cohen-Macaulayness of tangent cones of monomial curves in $\mathbb{A}^{4}(K)$, Preprint 2017, $21 \mathrm{pp}$, arXiv:1512.04204v4 [math.AC].

[7] V. Barucci, R. Fröberg, One-dimensional almost Gorenstein rings, J. Algebra 188 (1997), 418-442.

[8] V. Barucci, R. Fröberg, Associated graded rings of one-dimensional analytically irreducible rings, J. Algebra 304 (2006), 349-358.

[9] V. Barucci, R. Fröberg, M. Şahin, On free resolutions of some semigroup rings, J. Pure and Applied Algebra 218 (2014), 1107-1116.

[10] H. Bresinsky, Symmetric semigroups of integers generated by 4 elements, Manuscr. Math. 17 (1975), 205-219.

[11] H. Bresinsky, On prime ideals with generic zero $x_{i}=t^{n_{i}}$, Proc. Amer. Math. Soc. 47 no.2 (1975), 329-332.

[12] L. Bryant, Goto Numbers of a Numerical Semigroup Ring and the Gorensteiness of Associated Graded Rings, Comm. Algebra 38 (2010), 2092-2128.

[13] W. Bruns, J. Herzog, Semigroup rings and simplicial complexes, J. Pure Appl. Algebra 122 (1997), no. 3, 185-208.

[14] W. Bruns, J. Herzog, Cohen-Macaulay Rings, Revised Ed., Cambridge Stud. Adv. Math., vol. 39, Cambridge University Press, Cambridge, 1998.

[15] A. Campillo, C. Marijuan, Higher order relations for a numerical semigroup, Sém. Théor. Nombres Bordeaux (2) 3 (1991), no. 2, 249-260.

[16] M.P. Cavaliere, G. Niesi, On monomial curves and Cohen-Macaulay type, manuscripta math. 42 (1983), $147-159$.

[17] M. Cimpoeas, D.I. Stamate, On intersections of complete intersection ideals, J. Pure Appl. Algebra 220 (2016), 3702-3712.

[18] R. Conaway, F. Gotti, J. Horton, C. O'Neill, R. Pelayo, M. Williams, B. Wissman, Minimal presentations of shifted numerical monoids, Int. J. Algebra Comput. 28 (2018), 53-68.

[19] M. D'Anna, V. Micale, A. Sammartano, When the associated graded ring of a semigroup ring is Complete Intersection, J. Pure Appl. Algebra 217 (2013), 1007-1017.

[20] W. Decker, G.-M. Greuel, G. Pfister, H. Schönemann, Singular 3-1-6 - A computer algebra system for polynomial computations. http://www. singular.uni-kl.de (2012).

[21] M. Delgado, P. A. García-Sánchez, J. Morais, numericalsgps: a GAP package on numerical semigroups. (http://www.gap-system.org/Packages/numericalsgps.html).

[22] C. Delorme, Sous-monö̈des d'intersection complète de N, Ann. Sci. Ecole Norm. Sup. (4) 9 (1976), no. 1, 145-154.

[23] G. Denham, Short generating functions for some semigroup algebras, The Electronic Journal of Combinatorics 10 (2003), Paper R36.

[24] D. Eisenbud, Commutative Algebra with a View Toward Algebraic Geometry, Graduate Texts in Mathematics 150, Springer, 1995.

[25] D. Eisenbud, The Geometry of Syzygies, Graduate Texts in Mathematics 229, Springer, 2005.

[26] V. Ene, J. Herzog, Gröbner bases in commutative algebra, Graduate Studies in Mathematics 130, American Mathematical Society, 2012.

[27] K. Eto, Almost Gorenstein monomial curves in affine four space, J. Algebra 488 (2017), $362-387$.

[28] R. Fröberg, C. Gottlieb, R. Häggkvist, On numerical semigroups, Semigroup Forum 35 (1987), 63-83.

[29] R. Fröberg, On the homology of semigroup rings, slides for the Iberian Meeting on Numerical Semigroups-Porto 2008, available at http://cmup.fc.up.pt/cmup/ASA/numsgps_meeting/ slides/froberg.pdf.

[30] The GAP Group, GAP - Groups, Algorithms, and Programming, Version 4.7.5, 2014. (http: //www.gap-system.org). 
[31] A. Garcia, Cohen-Macaulayness of the associated graded of a semigroup ring, Comm. Algebra 10 (1982), no. 4, 393-415.

[32] I.García-Marco, J.L. Ramírez Alfonsín, O. Rødseth, Numerical semigroups II: pseudosymmetric AA-semigroups, J. Algebra 470 (2017), 484-498.

[33] P. Gimenez, I. Sengupta, H. Srinivasan, Minimal graded free resolutions for monomial curves defined by arithmetic sequences, J. Algebra 338 (2013), 294-310.

[34] P. Gimenez, H. Srinivasan, The structure of the minimal free resolution of semigroup rings obtained by gluing, Preprint 2017, arXiv:1710.07237 [math.AC], 19 pp.

[35] S. Goto, W. Heinzer, M. Kim, The leading ideal of a complete intersection of height two. II, J. Algebra 312 (2007), 709-732.

[36] D.R. Grayson, M.E. Stillman, Macaulay 2, a software system for research in algebraic geometry, available at http://www.math.uiuc.edu/Macaulay2/.

[37] J. Herzog, Generators and relations of Abelian semigroups and semigroup rings, Manuscripta Math. 3 (1970), 175-193.

[38] J. Herzog, When is a regular sequence super regular?, Nagoya Math. J. 83 (1981), 183-195.

[39] J. Herzog, T. Hibi, D.I. Stamate, The trace of the canonical module, Preprint 2016, arXiv:1612.02723 [math.AC], 38 pp.

[40] J. Herzog, M. E. Rossi, G. Valla, On the depth of the symmetric algebra, Trans. AMS 296 (1986), 577-606.

[41] J. Herzog, D.I. Stamate, On the defining equations of the tangent cone of a numerical semigroup ring, J. Algebra 418 (2014), 8-28.

[42] J. Herzog, D.I. Stamate, Quadratic numerical semigroups and the Koszul property, Kyoto J. Math. 57 (2017), 585-612.

[43] J. Herzog, K.-i. Watanabe, Almost symmetric numerical semigroups and almost Gorenstein semigroup rings, RIMS Kôkyûroku 2008 (2016), 107-120.

[44] R. Jafari, S. Zarzuela, Homogeneous numerical semigroups, Semigroup Forum (2018). https://doi.org/10.1007/s00233-018-9941-6.

[45] A.V. Jayanthan, H. Srinivasan, Periodic occurence of complete intersection monomial curves, Proc. Amer. Math. Soc. 141 (2013), 4199-4208.

[46] A. Katsabekis, Equations defining tangent cones of Gorenstein monomial curves, Preprint 2016, arXiv:1608.07100v1 [math.AC], 19 pp.

[47] J. Komeda, On the existence of Weierstrass points with a certain semigroup generated by 4 elements, Tsukuba J. Math. 6 (1982), 237-279.

[48] A. Kumar Roy, I. Sengupta, G. Tripathi, Minimal graded free resolution for monomial curves in $\mathbb{A}^{4}$ defined by almost arithmetic sequences, Comm. Algebra 45 (2017), 521-551.

[49] E. Kunz, The value-semigroup of a one-dimensional Gorenstein ring, Proc. Amer. Math. Soc. 25 (1970), 748-751.

[50] A. Marzullo, On the periodicity of the first betti number of the semigroup rings under translations, J. Ramanujan Math. Soc 28 (2) (2013).

[51] V. Micale, A. Olteanu, On the Betti numbers of some semigroup rings, Le Matematiche Vol. LXVII (2012) Fasc. I, 145-159.

[52] A. Moscariello, On the type of an almost Gorenstein monomial curve, J. Algebra 456 (2016), 266-277.

[53] H. Nari, T. Numata, K.-i Watanabe, Genus of numerical semigroups generated by three elements, J. Algebra 358 (2012), 67-73.

[54] H. Nari, Symmetries on almost symmetric numerical semigroups, Semigroup Forum 86 (2013) $140-154$.

[55] T. Numata, Almost symmetric numerical semigroups generated by four elements, Proc. Inst. Nat. Sci., Nihon Univ. 48 (2013), 197-207.

[56] T. Numata, A variation of gluing of numerical semigroups, Semigroup Forum 93 (2016), 152-160. 
[57] C. O'Neill, R. Pelayo, Apéry sets of shifted numerical monoids, Adv. Appl. Math. 97 (2018) $27-35$.

[58] A. Oneto, G. Tamone, Explicit minimal resolution for certain monomial curves, Preprint 2013, arXiv:1312.0789 [math.AC], 8 pp.

[59] A. Oneto, G. Tamone, Syzygies of GS monomial curves and Weierstrass property, Semigroup Forum 92 (2016), 258-273.

[60] I. Peeva, Graded Syzygies, Springer, 2011.

[61] J.L. Ramírez Alfonsín, The Diophantine Frobenius problem, Oxford Lecture Series in Mathematics and its Applications, 30, Oxford University Press, 2005.

[62] L. Robbiano, G. Valla, On the equations defining tangent cones, Math. Proc. Camb. Phil. Soc. 88 (1980), 281-297.

[63] J.C. Rosales, On presentations of subsemigroups of $\mathbb{N}^{n}$, Semigroup Forum 55 (1997), 152-159.

[64] J.C. Rosales, P.A. Garcia-Sánchez, Numerical Semigroups, Developments in Mathematics Series Vol 20, Springer, 2009.

[65] L. Sharifan, R. Zaare-Nahandi, Minimal free resolution of the associated graded ring of monomial curves of generalized arithmetic sequences, J. Pure Appl. Algebra 213 (2009), 360-369.

[66] L. Sharifan, R. Zaare-Nahandi, A class of monomial curves of homogeneous type, in: Extended Abstracts of the 22nd Iranian Algebra Seminar, 31th Jan-2nd Feb 2012, Hakim Sabzevari University, Sabzevar, Iran, 255-258.

[67] T. Shibuta, Cohen-Macaulayness of almost complete intersection tangent cones, J. Algebra 319 (2008), 3222-3243.

[68] D.I. Stamate, Asymptotic properties in the shifted family of a numerical semigroup with few generators, Semigroup Forum 93 (2016), 225-246.

[69] F. Strazzanti, A family of quotients of the Rees algebra and rigidity properties of local cohomology modules, Ph.D. Thesis, University of Pisa, 2016.

[70] B. Sturmfels, Gröbner Bases and Convex Polytopes, University Lecture Series 8, American Mathematical Society, 1996.

[71] M. Şahin, Extensions of Toric Varieties, The Electronic Journal of Combinatorics 18 (2011), Paper P93.

[72] M. Şahin, N. Şahin, On Pseudo Symmetric Monomial Curves, Comm. Alg. 46 (2018), 25612573.

[73] A. Tripathi, On a variation of the coin exchange problem for arithmetic progressions, Integers 3 (2003), A1, 5 pp.

[74] T. Vu, Periodicity of Betti numbers of monomial curves, J. Algebra 418 (2014), 66-90.

[75] K. Watanabe, Some examples of one dimensional Gorenstein domains, Nagoya Math. J. 49 (1973), 101-109.

[76] Ch. A. Weibel, An introduction to homological algebra, Cambridge Stud. Adv. Math., vol. 38, Cambridge University Press, Cambridge, 1994.

Dumitru I. Stamate, ICUB/Faculty of Mathematics and Computer Science, UniVersity of Bucharest, Str. Academiei 14, Bucharest, Romania

E-mail address: dumitru.stamate@fmi.unibuc.ro 